Portland State University

PDXScholar

3-1-2012

\title{
Power Characteristics of Homogeneously Broadened Index-Antiguided Waveguide Lasers
}

Chaofan Wang

Tsing-Hua Her

Lee W. Casperson

Portland State University

Follow this and additional works at: https://pdxscholar.library.pdx.edu/ece_fac

Part of the Electrical and Computer Engineering Commons

Let us know how access to this document benefits you.

\section{Citation Details}

Chaofan Wang, Tsing-Hua Her, Lee W. Casperson . Power characteristics of homogeneously broadened index-antiguided waveguide lasers. Optics Letters, Volume 37, Number 5 (March 2012), pp. 815-817

This Article is brought to you for free and open access. It has been accepted for inclusion in Electrical and Computer Engineering Faculty Publications and Presentations by an authorized administrator of PDXScholar. Please contact us if we can make this document more accessible: pdxscholar@pdx.edu. 


\title{
Power characteristics of homogeneously broadened index-antiguided waveguide lasers
}

\author{
Chaofan Wang, ${ }^{1}$ Tsing-Hua Her, ${ }^{1, *}$ and Lee W. Casperson ${ }^{2}$ \\ ${ }^{1}$ Department of Physics and Optical Science, and Center for Optoelectronics and Optical Communications, \\ University of North Carolina at Charlotte, 9201 University City Boulevard, Charlotte, North Carolina 28223, USA \\ ${ }^{2}$ Department of Electrical and Computer Engineering, University of North Carolina at Charlotte, \\ 9201 University City Boulevard, Charlotte, North Carolina 28223, USA \\ *Corresponding author: ther@uncc.edu
}

Received November 4, 2011; accepted December 20, 2011;

posted December 22, 2011 (Doc. ID 157478); published February 21, 2012

A model is reported that describes a bidirectional homogeneously broadened index-antiguided (IAG) slab laser having arbitrary single-pass gain and distributed losses. Maximum extraction efficiency and corresponding optimum output coupling are determined for various values of unsaturated gain and loss per pass. A method is proposed to determine the intrinsic laser parameters from output power measurements. (c) 2012 Optical Society of America OCIS codes: $\quad 060.2320,230.7400$.

Index-antiguided (IAG) waveguide lasers have been known for many years. They were introduced as step-index hollow-dielectric antiguided waveguide lasers [1,2] and graded-gain dispersion-antiguided waveguide lasers [3]. Because these were low-pressure small-activevolume gas lasers, output power levels were modest. Recently, there has been increasing interest in the high-power capabilities of solid IAG-waveguide lasers $[4,5]$. With the core index of refraction being lower than the cladding, this configuration has the potential for good transverse mode discrimination leading to single-mode amplification. Although encouraging results for singletransverse-mode operation in IAG fibers with core diameters up to $400 \mu \mathrm{m}$ [6] and a slope efficiency of 4.5\% [7] have been experimentally demonstrated, a complete analysis of high-gain IAG-waveguide laser oscillators is still lacking. In this Letter, we report a theoretical investigation of the power and efficiency characteristics of IAGwaveguide oscillators. The results described here include accurate semianalytic models for the design of saturating IAG-waveguide oscillators with arbitrary gain. Quantitative results are presented that relate maximum power extraction efficiency and optimum output coupling over a wide range of laser parameters.

Previously we have obtained detailed numerical solutions for the fields in IAG-waveguide amplifiers using the beam propagation method (BPM), and we also developed much simpler but highly accurate approximate semianalytical solutions $[8,9]$. We showed that, for practical designs of IAG waveguides, the transverse field distribution within the core of a slab-geometry IAG-waveguide amplifier is closely described by a cosine function having a zero at the core boundary. We defined the power in the core $P_{c}$ per unit length along the unbounded dimension by integrating intensity along the bound dimension. Then the normalized average intensity in the core $I_{c}^{\prime}(z)$, defined by $I_{c}^{\prime}(z)=P_{c}(z) /\left(d \cdot I_{s}\right)$, where $I_{s}$ is the saturation intensity and $d$ is the width of the core, follows the following ordinary differential equation in a homogeneously broadened gain medium []ㅛ:

$$
\frac{d I_{c}^{\prime}}{d z}=g_{o} I_{c}^{\prime}\left\{\frac{1}{I_{c}^{\prime}}\left[1-\frac{1}{\left[1+2 I_{c}^{\prime}\right]^{1 / 2}}\right]\right\}-\alpha I_{c}^{\prime}
$$

where the unsaturated gain coefficient $g_{o}$ is assumed to be independent of $z$ (uniform pumping), and $\alpha$ is the distributed loss coefficient. Note that the term in braces represents the saturation factor.

Eq. (1) has been shown to be useful for finding the amplification and saturation of the propagating fundamental mode in a homogeneously broadened slab IAG-waveguide amplifier [9]. To generalize this one-directional amplifier equation to a bidirectional laser oscillator with the cavity extending from $z=0$ to $z=L$, we modify only the form of the saturation factor to include saturation by both the left and right propagating waves. The right and left propagating intensities in a homogeneously broadened IAG-waveguide oscillator are thus governed by the following set of two coupled first-order nonlinear ordinary differential equations:

$$
\frac{d I_{c}^{+}}{d z}=g_{o} I_{c}^{++}\left\{\frac{1}{I_{c}^{++}+I_{c}^{\prime-}}\left[1-\frac{1}{\left[1+2\left(I_{c}^{+}+I_{c}^{\prime-}\right)\right]^{1 / 2}}\right]\right\}-\alpha I_{c}^{\prime+},
$$

$$
\begin{aligned}
\frac{d I_{c}^{\prime-}}{d z}= & -g_{o} I_{c}^{+}\left\{\frac{1}{I_{c}^{\prime+}+I_{c}^{\prime-}}\left[1-\frac{1}{\left[1+2\left(I_{c}^{\prime+}+I_{c}^{\prime-}\right)\right]^{1 / 2}}\right]\right\} \\
& +\alpha I_{c}^{-},
\end{aligned}
$$

subject to the boundary conditions imposed by the reflectors at the amplifier ends

$$
I_{c}^{++}(0)=R_{l} I_{c}^{-}(0), \quad I_{c}^{-}(L)=R_{r} I_{c}^{+}(L),
$$

where $R_{l}$ and $R_{r}$ are the mirror reflectances at the left-hand $(z=0)$ and the right-hand $(z=L)$ ends of the laser, respectively; the minus and plus superscripts refer to the fields propagating toward the left and right ends, respectively.

The saturation equations for an antiguided bidirectional laser amplifier, Eqs. (2) and (3) above, are more complicated than the corresponding uniform-plane-wave results. On the other hand, for small values of the saturating intensities, the saturation factors in Eqs. (2) and (3) reduce to unity, and in this limit the saturation equations 
may be approximated by the plane-wave forms for an unsaturated laser:

$$
\frac{d I_{c}^{\prime \pm}(z)}{d z}= \pm\left(g_{o}-\alpha\right) I_{c}^{ \pm}(z)
$$

At threshold, Eq. (5) can be integrated subject to the boundary conditions at the laser mirrors to obtain the familiar result for the threshold gain $g_{\mathrm{th}}$ :

$$
g_{\mathrm{th}}=\alpha-\frac{1}{L} \ln \left(R_{l} R_{r}\right) .
$$

For arbitrary levels of saturation, one has to solve Eqs. (2)-(4) self-consistently. Notice that if the left-hand side of Eq. (3) is divided by $I_{c}^{++}(z)$ and the left-hand side of Eq. (4) is divided by $I_{c}^{\prime-}(z)$, then the right-hand sides of these equations differ from each other only by a minus sign. The solution of this equation is known as the first integral:

$$
I_{c}^{\prime+}(z) I_{c}^{-}(z)=a,
$$

where $a$ is a constant to be determined, indicating that the product of the right and left propagating intensities is a constant over the length of the amplifier. With the boundary conditions given in Eq. (4), the left propagating intensities can be eliminated in Eq. (7), and the result can be written

$$
a=\frac{1}{R_{l}}\left(I_{c}^{\prime+}(0)\right)^{2}=R_{r}\left(I_{c}^{\prime+}(L)\right)^{2} .
$$

Equation (7) can also be used to eliminate $I_{c}^{-}(z)$ in Eq. (2) to yield

$$
\begin{aligned}
\frac{d I_{c}^{+}}{d z}= & g_{o} I_{c}^{++}\left\{\frac{1}{I_{c}^{++}+\frac{a}{I_{c}^{+}}}\left[1-\frac{1}{\left[1+2\left(I_{c}^{\prime+}+\frac{a}{I_{c}^{+}}\right)\right]^{1 / 2}}\right]\right\} \\
& -\alpha I_{c}^{+} .
\end{aligned}
$$

Equations (8) and (9) provide a complete set for solving for the intensities of the right and left propagating waves anywhere inside the homogeneously broadened IAG-waveguide oscillator. Equation (9) is considerably more complicated than its plane-wave counterpart $[\underline{10}, 11]$, but can be solved numerically. For a common laser configuration where $R_{l}=1$ at $z=0$ (perfectly reflecting), the output normalized intensity $I^{\prime}$ outside the output coupler can be calculated according to $I^{\prime}=$ $\left(1-R_{r}\right) I_{c}^{\prime+}(L)$. From this result the extraction efficiency $\eta$ of the IAG laser, assuming the waveguide is uniformly pumped to have a constant unsaturated gain coefficient $g_{o}$ over the entire cross section and the length $L$, can be defined by

$$
\eta=\frac{I^{\prime}}{g_{0} L}
$$

The denominator $g_{0} L$ is the unsaturated gain per pass and represents the maximum normalized optical inten- sity available from the population inversion. Such a value could be obtained in a plane-wave laser resonator exhibiting no distributed loss and nearly $100 \%$ output coupler reflectance [11].

The theoretical model we have developed can be used to design and optimize IAG-waveguide lasers. For this purpose it is convenient to solve Eq. (9) in terms of the unsaturated gain per pass $g_{o} L$ and loss per pass $\alpha L$. For a given set of $g_{0} L$ and $\alpha L$, there exists an optimized output coupler reflectance $R_{\text {opt }}$ to obtain a maximum extraction efficiency $\eta_{\max }$. Figure 1 shows $\eta_{\max }$ versus its corresponding $R_{\text {opt }}$ for various values of $g_{0} L$ and $\alpha L$. Note that Fig. 1 is composed of only 200 data points with lines jointing them so that the smoothness of curves can be improved if more points are sampled. The optimum parameters for a given laser configuration are represented by the intersecting point of the two curves with the corresponding values of $g_{o} L$ and $\alpha L$. Very high extraction efficiency is only attainable with very low loss and nearly $100 \%$ output coupler reflectance. As loss increases, $\eta_{\max }$ decreases significantly. Figure 1 indicates that high gain and low loss are desirable for IA $\bar{G}$ oscillators because they lead to not only higher $\eta_{\max }$, but also more robust laser operation as $\eta_{\max }$ becomes less sensitive to variation of loss and mirror reflectance.

The above trend is qualitatively similar to that of a plane-wave oscillator [11], and one might be tempted to use the simpler plane-wave saturation model to design IAG oscillators. To compare their performance, we consider a plane-wave oscillator with a finite aperture equal to that of the IAG oscillator. Figure 2 shows the relative efficiency $\eta_{r}$, defined by the ratio of $\bar{\eta}_{\text {max }}$ of an IAG oscillator to the corresponding $\eta_{\max }$ of a plane-wave oscillator, as a function of $g_{0} L$ between $g_{\mathrm{th}} L$ to 10 for various values of $\alpha L$. Note that $\eta_{r}$ is always less than unity. Physically, this is due to the cosine-shaped mode field of the IAG slab waveguide, which leads to nonuniform gain saturation along the transverse dimension, whereas a plane-wave mode field saturates uniformly across the entire aperture. This, however, does not imply that an IAG oscillator operated in the fundamental mode is less efficient, as a plane-wave oscillator with a mode

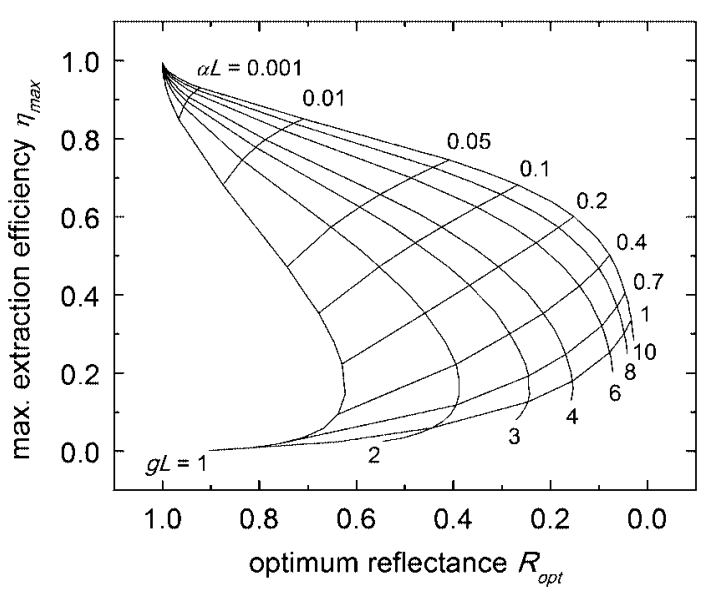

Fig. 1. Maximum extraction efficiency $\eta_{\max }$ versus optimum output coupler reflectance $R_{\text {opt }}$ of homogeneously broadened IAG slab lasers for various values of $g_{0} L$ and $\alpha L$. 


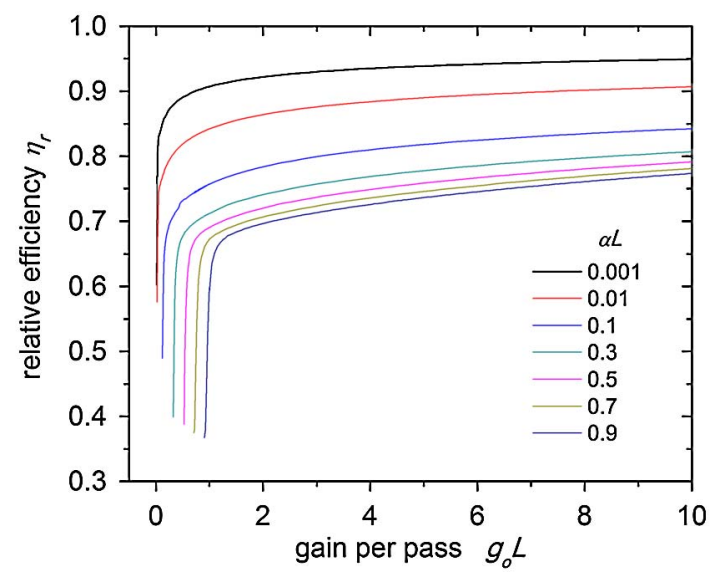

Fig. 2. (Color online) Dependence of the ratio $\eta_{r}$ of $\eta_{\max }$ of IAG slab oscillators to that of plane-wave oscillators on $g_{0} L$ for different $\alpha L$.

field filling a finite aperture cannot be realized in singlemode operation. Figure 2 shows that all curves have similar trends: $\eta_{r}$ is low and rises sharply right above the threshold, after which it increases smoothly as gain increases. In addition, lower loss leads to higher $\eta_{r}$. These trends can be understood qualitatively by noting that, as gain increases or loss decreases, the transverse gain in the IAG oscillator becomes more uniformly saturated across the aperture, leading to higher $\eta_{r}$. In the theoretical limit of no loss, i.e., distributed loss $=0$ and nearly $100 \%$ mirror reflectance where the IAG oscillator is nearly fully saturated transversely, $\eta_{\max }$ of an IAG oscillator approaches $100 \%$ (see Fig. 1), which is comparable to that of a plane-wave oscillator [12]. Figure 2 shows that the prediction from a plane-wave saturation model is too optimistic and is strongly dependent on gain or loss. Therefore, Eq. (9) would provide better results when designing and analyzing IAG oscillators.

Experimental determination of the three intrinsic laser parameters $g_{o}, \alpha$, and $I_{s}$ would also be useful. Assume the slab laser has a mode size $l$ along the unbounded dimension, which could be defined by the width of the optical or electrical pumping. The power extracted from a homogeneously broadened IAG slab laser is then

$$
P=I_{s} \cdot d \cdot l \cdot I^{\prime}\left(g_{o}, \alpha, R\right)
$$

The above equation has three unknowns $\left(g_{o}, \alpha, I_{s}\right)$ if the mirror reflectance $R$ is known and therefore requires three measurements to obtain its solution. Let the mirror reflectance and its corresponding output power of each measurement $i$ be $R_{i}$ and $P_{i}$, respectively, for $i=1$ to 3 . For each $R_{i}$, Eq. (11) indicates

$$
\frac{I_{i}^{\prime}\left(g_{o}, \alpha\right)}{P_{i}}=\left(I_{s} \cdot d \cdot l\right)^{-1}, \quad i=1 \text { to } 3
$$

For plane-wave oscillators, the solution to Eq. (12) can be obtained analytically [11], whereas for IAG oscillators this solution must be obtained numerically. One potential approach is to define a function $z(x, y) \equiv I^{\prime}(x, y) / P$, where $x$ and $y$ are variables of gain and loss coefficients, respectively. For each $i, z_{i}$ represents a curved surface in the three-dimensional space of $(x, y, z)$. These three surfaces can be obtained numerically by solving Eqs. (8) and (9) for each of the three pairs of $R_{i}$ and $P_{i}$. Since the laser intrinsic parameters satisfy the three equations in Eq. (12) simultaneously, they can be obtained from the intersection point of the three curved surfaces $z_{i}$, whose coordinates are $\left(g_{o}, \alpha,\left(I_{s} \cdot d \cdot l\right)^{-1}\right)$.

In conclusion, a formalism has been described for determining the distributions and amplitudes of the fields in bidirectional slab-geometry IAG laser oscillators with homogeneous line broadening. Detailed analysis shown here provides extraction efficiency and optimum choice of output coupler for arbitrary laser parameters. Our results are significantly different from those obtained assuming a conventional plane-wave analysis.

The authors are pleased to acknowledge support from the National Science Foundation under grant 0925992. T. Her also acknowledges the financial support from a DARPA Young Faculty Award (HR0011-08-1-0065). The authors note with sadness the recent passing of A. E. Siegman, who initiated the subject being studied and reported on in this paper. One of us (LWC) also recalls numerous enjoyable and stimulating discussions with Dr. Siegman over a period of more than 40 years. He will be missed.

\section{References}

1. E. A. J. Marcatili and R. A. Schmeltzer, Bell Syst. Tech. J. 43, 1783 (1964).

2. P. W. Smith, Appl. Phys. Lett. 19, 132 (1971).

3. L. W. Casperson and A. Yariv, Appl. Opt. 11, 462 (1972).

4. A. E. Siegman, J. Opt. Soc. Am. A 20, 1617 (2003).

5. A. E. Siegman, Y. Chen, V. Sudesh, M. C. Richardson, M. Bass, P. Foy, W. Hawkins, and J. Ballato, Appl. Phys. Lett. 89, 251101 (2006).

6. Y. Chen, V. Sudesh, T. McComb, M. C. Richardson, M. Bass, and J. Ballato, J. Opt. Soc. Am. B 24, 1683 (2007).

7. R. Sims, V. Sudesh, T. McComb, Y. Chen, M. Bass, M. Richardson, A. G. James, J. Ballato, and A. Siegman, in OSA Technical Digest Series (CD) (Optical Society of America, 2009), WB3.

8. T.-H. Her, X. Ao, and L. W. Casperson, Opt. Lett. 34, 2411 (2009).

9. C. Wang, T.-H. Her, L. Zhao, X. Ao, L. W. Casperson, C.-H. Lai, and H.-C. Chang, J. Lightwave Technol. 29, 1958 (2011).

10. W. Rigrod, IEEE J. Quantum Electron. 14, 377 (1978).

11. G. Schindler, IEEE J. Quantum Electron. 16, 546 (1980).

12. N. Hodgson and H. Weber, Laser Resonators and Beam Propagation: Fundamentals, Advanced Concepts and Applications, 2nd ed. (Springer, 2005). 